\title{
Touch- and brush-spinning of nanofibers
}

Alexander Tokarev ${ }^{1}$, Darya Asheghali ${ }^{1}$, Ian. M. Griffiths ${ }^{2}$, Oleksandr Trotsenko ${ }^{1}$, Alexey Gruzd $^{1}$, Xin Lin ${ }^{1}$, Howard A. Stone ${ }^{3}$, Sergiy Minko ${ }^{1, *}$

${ }^{1}$ Nanostructured Materials Laboratory, University of Georgia, Athens, GA, 30602, USA

${ }^{2}$ Mathematical Institute, University of Oxford, Oxford, OX2 6GG, UK

${ }^{3}$ Department of Mechanical and Aerospace Engineering, Princeton University, Princeton, NJ, 08544, USA

*e-mail: sminko@uga.edu

Polymer nanofibers are used in numerous applications ranging from the design of new composite materials to the fabrication of nanostructured biomimetic scaffolds for artificial bones and organs for regenerative medicine. Here, we describe a simple controllable set-up for drawing single filament nanofibers from polymer solutions or melts using a rotating rod or a set of rods (round brush). The set-up can be assembled in a few minutes and applied to fabricate customized nanofiber scaffolds and meshes for various applications. The resulting fiber diameter is controlled precisely in the range $40 \mathrm{~nm}$ to $5 \mu \mathrm{m}$ by adjusting the rotational speed and polymer concentration. Owing to the simple design and capability to manipulate single nanofibers, the spinning set-up can be used to wind a single filament into unidirectional, orthogonal or randomly oriented $2 \mathrm{D}$ and 3D meshes with controlled density, thickness and combinations of different fibers and materials in the scaffolds. The method is scalable and can be implemented easily for laboratory and industrial manufacturing.

In the past decade polymer nanofibers have found applications in many different areas such as bone and tissue regeneration $(1,2)$, biosensors $(3)$, fuels cells $(4,5)$, design of composites $(6)$ and polymer nanofiber films (7). Water/air purification systems, personal care products and membranes utilize the high surface area of nanofibers to enhance transport and filtration properties (8-10) while nanofibers with tunable conductivity and molecular memory are promising building blocks for miniaturized devices (11). Currently electrospinning is the most popular method for nanofiber production (12). In this method a droplet of polymer solution is stretched to form a fiber by a high-voltage electric field. Electrospinning requires high voltages (20-30 kV) and depends strongly on the dielectric properties of materials (12) that call for adjustment of spinning solutions and experienced operators of the spinning set-up. Fibers are deposited on collectors (plane electrodes or frames) or rotating electrodes of a cylindrical shape for the fabrication of 2D and 3D fibrous nonwoven structures (13).

The recent discovery of the ability of nanofibers to help to form bones and tissues in combination with stem cells $(14,15)$ has resulted in a significantly increased interest in the development of simple methods for nanofiber fabrication that can be conducted in biological and biomedical laboratories. However, problems of antigenicity and immunogenicity of a donor's biological materials, as well as specific needs in the shapes, dimensions and morphologies of tissue implants call for the fabrication of customized scaffolds $(16,17)$ that can be engineered and fabricated at a health-provider facilities.

The simplest method of nanofiber fabrication is direct drawing from a polymer solution using a glass micropipette (18). This method however was not scaled up and thus did not find practical applications (19). Here, we introduce a scalable method of nanofiber spinning named touchspinning. A glass rod $(0.3 \mathrm{~mm}$ to a few $\mathrm{mm}$ in diameter $)$ is glued to a rotating stage, whose diameter 
can be chosen over a wide range of a few $\mathrm{cm}$ to more than one meter (Fig. 1A). A polymer solution is supplied, for example, from the needle of a syringe pump that faces the glass rod. The distance between the droplet of polymer solution and the tip of the glass rod is adjusted so that the glass rod contacts the polymer droplet as it rotates (Fig. 1B). Following the initial "touch", the polymer droplet forms a liquid bridge. As the stage rotates the bridge stretches and the fiber length increases, while the diameter decrease due to mass conservation (Fig. 1C). The surface area of the liquid thread increases with its elongation and thus generates a larger surface area for eventual solvent evaporation from the thread. For a polymer melt, the fiber drawing is followed by cooling and crystallization of the polymer.
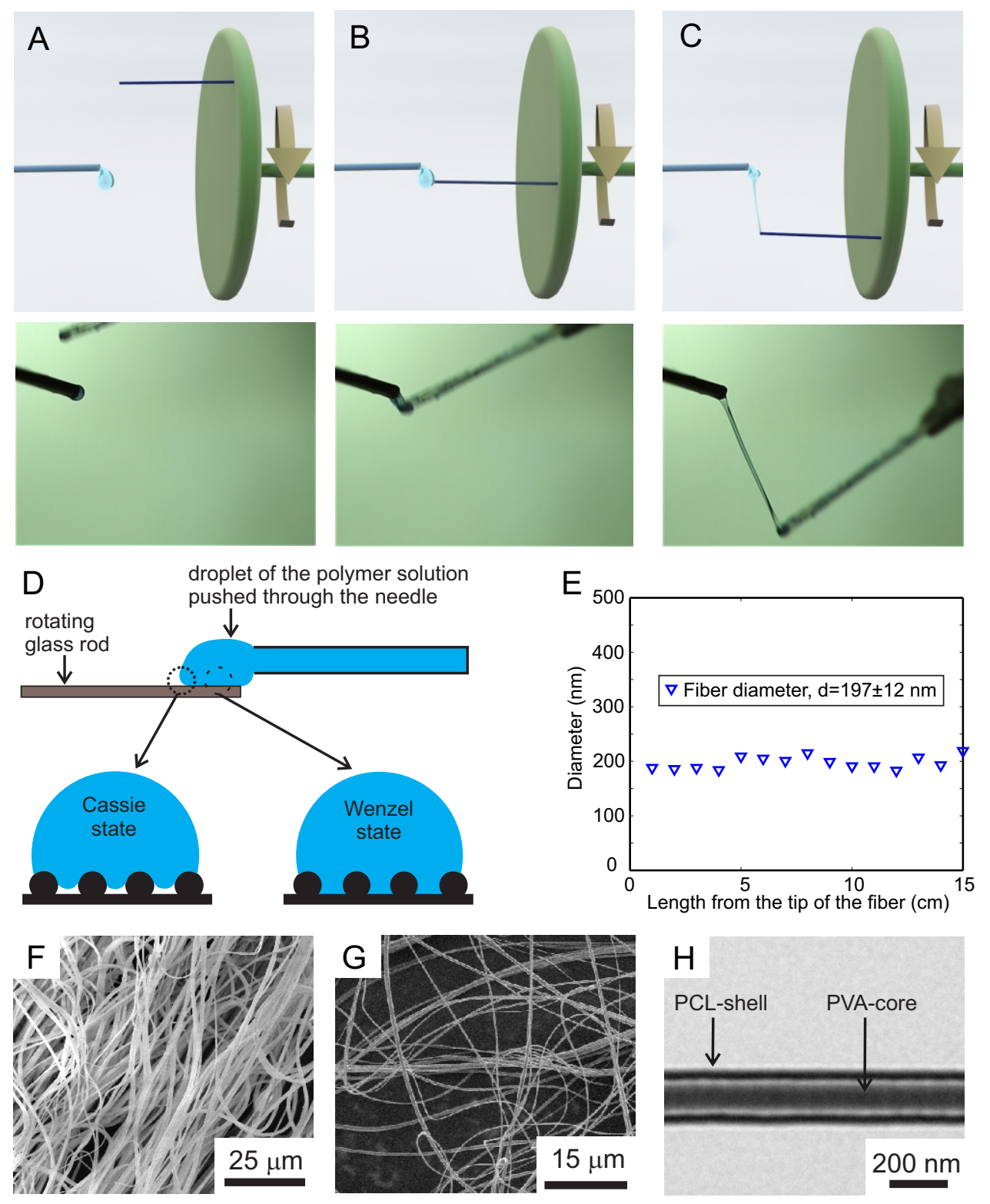

Fig. 1. Touch-spinning of nanofibers. (A) A glass rod is glued to a rotating stage and a droplet of the polymer solution is pushed through the needle with an automated pump. (B) The rotating glass rod touches the droplet of the polymer solution. (C) The glass rod continues rotation and a liquid bridge is formed between the glass rod and the needle tip. The liquid bridge solidifies upon solvent evaporation (or cooling for polymer melts) and fibers are collected along the edge of the rotating stage on mounted bars (not shown here). (D) Rotating glass rod touches the polymer solution droplet 
at a high speed. The local pressure overcomes the breakthrough pressure and results in transition to the Wenzel complete wetting while further spreading of liquid outside the high pressure area is limited by partial wetting and non-wetting Cassie states. (E) Measurements of the fiber diameter along a $15 \mathrm{~cm}$ length. (F) SEM image of PEO nanofibers. (G) SEM image of Teflon nanofibers. (H) TEM image of core-shell PVA-PCL nanofiber.

The touch-spinning set-up was used for drawing polyethylene oxide (PEO) fibers from aqueous solutions. However, after several revolutions a large amount of dried polymer accumulated on the surface of the glass rod due to wetting of the rod by the PEO solutions and its subsequent spreading; as the PEO droplets solidify on the glass rod fiber drawing becomes impossible. In order to avoid this problem we coated the rod with $1 \mathrm{H}, 1 \mathrm{H}, 2 \mathrm{H}, 2 \mathrm{H}$-perfluorodecyltriethoxysilane. For the fluorosilane surface-modified rod the advancing contact angle was measured to be $\Theta_{\mathrm{a}}=118^{\circ}$ for water and $\Theta_{\mathrm{a}}=107^{\circ}$ for a $3.5 \%$ PEO solution, while the receding contact angles were $\Theta_{\mathrm{r}}=98^{\circ}$ and $\Theta_{\mathrm{r}}=75^{\circ}$, respectively (Fig. S1, S2 in Supplementary Materials (SM)). Even though the modified glass rod repelled water, the set-up produced nanofibers because the modified surface adhered to the polymer solution droplet due to the wetting hysteresis. The modification with fluorosilane aids in avoiding accumulation of the polymer on the glass rod surfaces due to non-wetting or partial wetting, thus providing conditions for excellent reproducibility of fiber drawing due to the selfcleaning properties of the rod. The same results were obtained for superhydrophobic and superoleophobic coatings of the rod surface (see SM). The pressure $\left(\sim 10^{6} \mathrm{~Pa}\right)$ generated when the rotating rod hits the droplet is much greater than the breakthrough pressure for typical composite surfaces with a re-entrant geometry $\left(\sim 10^{3} \mathrm{~Pa}\right)(20)$. This results in a transition from Cassie to the fully wetted Wenzel state (Fig. 1D), causing wetting hysteresis. For polymer solutions in aqueous and organic solvents, all of the coatings were found to have no build-up of polymer layers on the surface of the rod. Our results demonstrate that the touch-spinning method can be realized when the wetting behavior of the rod is optimized to non-wetting or partial wetting conditions with wetting hysteresis.

Examples of touch-spun PEO nanofibers are shown in the SEM image in Fig. 1F. The resulting fibers are very close to homogeneous in diameter along the fiber length (Fig. 1E). This method is not sensitive to the dielectric properties of the polymer solutions and thus can be used to draw a range of fibers from various polymer solutions and melts. For example, Teflon nanofibers can be fabricated by electrospinning only if blended with another polymer $(21,22)$ since Teflon is only soluble in liquids with low dielectric constants. Here we used the touch-spinning method to produce Teflon nanofibers from a solution of TAF 1600 (copolymer of 2,2-bis(trifluoromethyl)-4,5-difluoro-1,3dioxole) in Fluorinert ${ }^{\circledR}$ FC-40 fluid with dielectric constant 1.9 (Fig. 1G). The touch-spinning method can also be used for drawing fibers from polymer melts. For example, touch-spun polyethylene microfibers were drawn from polyethylene (PE) melted by a heat gun at $150^{\circ} \mathrm{C}$ (see SM for an optical microscope image of a PE fiber). We used the touch-spinning method to produce core-shell PVA-PCL nanofibers (Fig. 1H).

In the touch-spinning method, the resulting nanofiber diameter can be varied by changing the rotation speed of the stage or the concentration of the polymer solution. Fig. 2A summarizes variations in fiber diameter as a function of PEO concentration in the range from 3.5-9 wt $\%$ and as a function of rotational speed in the range 500-2500 RPM: the fiber diameter decreases with increasing rotational speed and decreasing polymer concentration. The error bars show the standard deviation of the fiber diameter in a series of experiments, and are comparable with those for fibers 
made using other traditional nanofiber spinning methods $(23,24)$. In each series the standard deviation was measured for one hundred $15 \mathrm{~cm}$ long fibers in different samples of the same batch along the fiber's length. For example, the standard deviation for $125 \mathrm{~nm}$ fibers is $\pm 36 \mathrm{~nm}$ (all values are presented in Fig. 2A as error bars and in SM, Table S3).
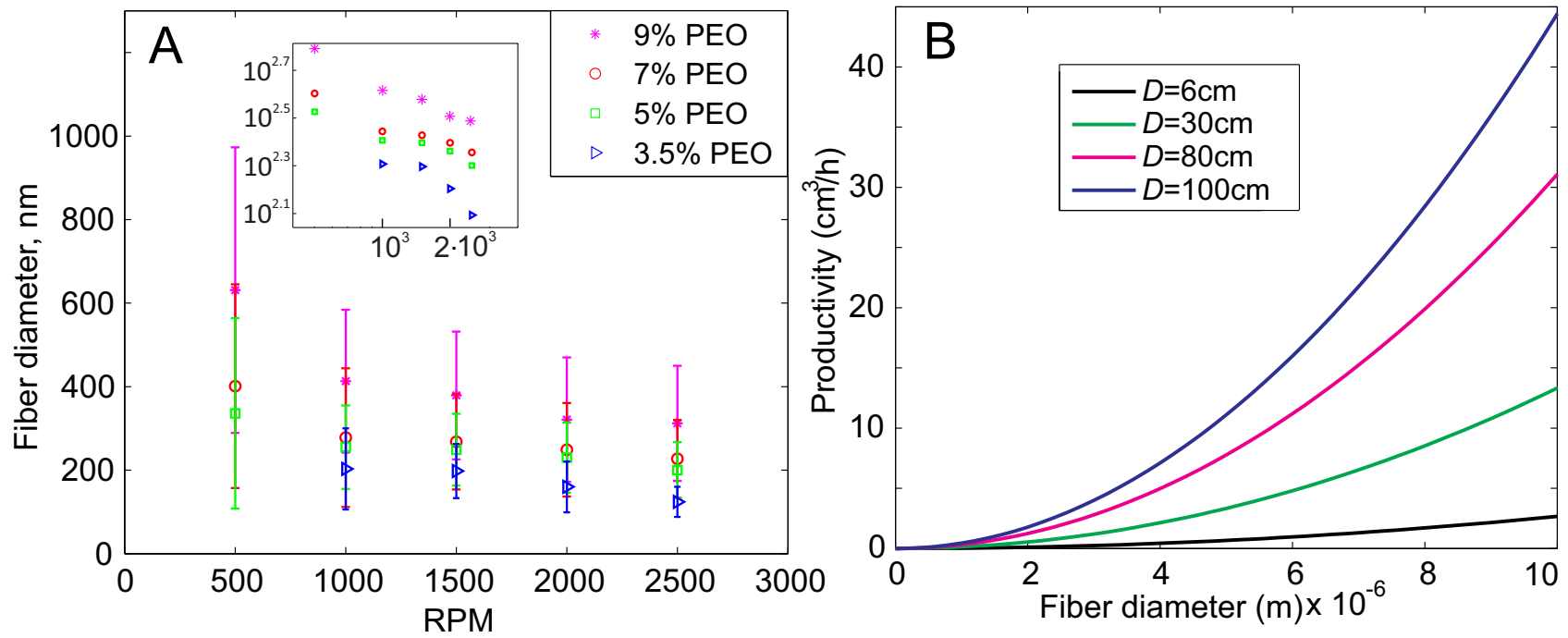

Fig. 2. Controlling the diameter of fibers produced by touch-spinning. (A) Diameters of touchspun PEO nanofibers at different rotational speeds. Inset shows graph A with log-log axes. (B) Productivity of touch-spinning, where $D$ is the diameter of the rotating stage.

A series of experiments with different diameters of glass rods (from $0.33 \mathrm{~mm}$ to $1.8 \mathrm{~mm}$ ) and different wetting properties of the surface of the rods (water contact angle from $0^{\circ}$ to $160^{\circ}$ ) demonstrated no effect on the fiber diameter, which indicates that the fiber diameter is a function of the interplay of parameters for stretching of the polymer liquid formed between the surface supplying the polymer liquid and the tip of the glass rod, as discussed below. The wetting behavior of the rotating rod is critical to avoid contamination of the rod with polymer deposits and has no effect on the fiber diameter (see SM for details). The rate of production of fibers in touch-spinning $\left(V_{p}, \mathrm{~cm}^{3} / h\right)$ clearly depends on the diameter of the rotating stage $\left(D_{s}\right)$, rotational speed $(\omega)$ and fiber radius $\left(R_{\mathrm{f}}\right)-V_{\mathrm{p}}=\pi R_{\mathrm{f}}^{2}\left(\pi D_{s} \omega\right)$. The productivity of the method estimated for a single rod is in the same range as for electrospinning (23) (Fig. 2B).

A key advantage of the touch-spinning process is its demonstrable ability to generate fibers with a uniform diameter (Fig. 1E). This feature arises as a result of the drawing process, which thins the fiber via two distinct mechanisms: axial stretching due to the rotation of the stage and capillarity, which acts to drain fluid from the fiber back into the source droplet. The draw-out process sufficiently far from break-up may be described by exploiting the slenderness of the fiber (17) to write simplified time-dependent, one-dimensional governing equations for a mass balance, including solvent evaporation, and an axial linear momentum balance:

$$
\frac{\partial}{\partial t}\left(R^{2}\right)+\frac{\partial}{\partial z}\left(R^{2} \omega\right)=-\alpha R, \rho R^{2}\left(\frac{\partial \omega}{\partial t}+\omega \frac{\partial \omega}{\partial z}\right)=\frac{\partial}{\partial z}\left(3 \mu R^{2} \frac{\partial \omega}{\partial z}\right)+\gamma \frac{\partial R}{\partial z}(1 a, b)
$$

Here $R$ is the fiber radius, $w$ the fluid velocity, $z$ the axial position along the fiber, $\rho, \gamma$ and $\eta$ the fluid density, surface tension and viscosity respectively, and $\alpha$ is a coefficient characterizing the evaporation rate. 
For the touch-spinning operating regimes the reduced capillary number, $\varepsilon \mathrm{Ca}=\mu W / \gamma<<1$, where $\varepsilon$ is the typical fiber aspect ratio of diameter to length, and $W$ is the speed of the rod at the tip. In this limit, Eq. ( $1 b)$ reduces to $\partial R / \partial z=0$ and so $R=R(t)$ only. This result indicates that surface tension will act to smooth out any axial variations in radius and hence we expect to observe uniform thinning for the bulk of the fiber, with variations in the fiber radius constrained to a small boundary layer of order $\varepsilon \mathrm{Ca}$ times the fiber length near the droplet end. Within this end boundary layer, surface-tension effects are important and capillary suction will act to drain liquid from the bulk fiber into the droplet (18). Consequently, following drawing the resulting fiber will be approximately uniform along its entire length.

The drawing process takes place on a short (millisecond) timescale: following fracture of the fiber, drawing ceases. Thereafter the fiber diameter decreases on a longer timescale (of the order of seconds) due to evaporation. In the first stage the polymeric fluid undergoes significant strains, on the order of the square of the ratio of the initial to final fiber diameters. Under such strains, the viscosities of polymeric fluids become very large (19), and so under the short drawing timescale the polymers within the fluid will have insufficient time to reorganize. In Eq. (1) this corresponds to a dependence of viscosity $\mu$ on extension rate, $\partial w / \partial z$. Due to the uniform nature of the fiber shape during the drawing stage before fracture, the fiber will also continue to thin uniformly during the evaporation stage. As a result, both the drawing and evaporation stages ensure that uniform fibers are generated, as observed in Fig. 1 and as also found in other spinning processes.

The diameter of the fibers fabricated by the touch-spinning process may be tailored in a variety of simple ways as we have shown, for example, by adjusting the speed of the top of the rod, $W$, or the polymer concentration (Fig. 2A). We find that the empirically observed fiber diameter $R$ that results from touch-spinning obeys an approximate power-law relation $R \sim W^{-\beta}$, where $\beta<1 / 2$. This result implies a weaker dependence of fiber diameter on rotation speed than in the case of steady fiber drawing in the absence of evaporation (in which case $\beta=1 / 2$ ). This reduced dependence may be rationalized by the fact that, although the fiber diameter is reduced with increasing draw speed, the volume of fluid taken up by the glass rod during contact increases with rotation rate thus diminishing the strength of the power law (see, for example, (20), which documents how the extracted volume depends on the speed of withdrawal for a model geometry).

The final fiber diameter is also observed to increase with concentration of PEO (Fig. 2A), which we identify with an increase in viscosity. This result may be attributed to the fact that a viscosity increase raises the axial viscous stresses, which impedes the stretching. We note that, as documented in (21), the inclusion of evaporation in Eq. (1a) also adjusts the value of $\beta$.

We have also demonstrated the simplicity and scalability of this new touch-spinning method by using a round hairbrush composed of the order of 600 filaments (Fig. 3A). The brush was attached to an electrical motor via the brush grip. The set-up was fed by a PEO solution poured onto a Teflon film placed underneath the round brush so that the brush filaments touched the droplets of the polymer solution but did not scratch the film. (In Fig. 3A PEO solution is mixed with green dye for visualization purposes.) Nanofibers were brush-spun from the free-liquid surface with a rotating hair brush. Figs. 3B and C show the $200 \mathrm{~nm}$ nanofibers produced in 1 and 5 minutes respectively at 3000 RPM, and an SEM image of the resulting fibers is shown in Fig. 3D. The total length of the fibers produced by the 600 -filament brush with $D=60 \mathrm{~mm}$ at $3000 \mathrm{RPM}$ in 5 minutes is $1700 \mathrm{~km}$, which is a sufficient amount for a typical tissue-engineering experiment. 


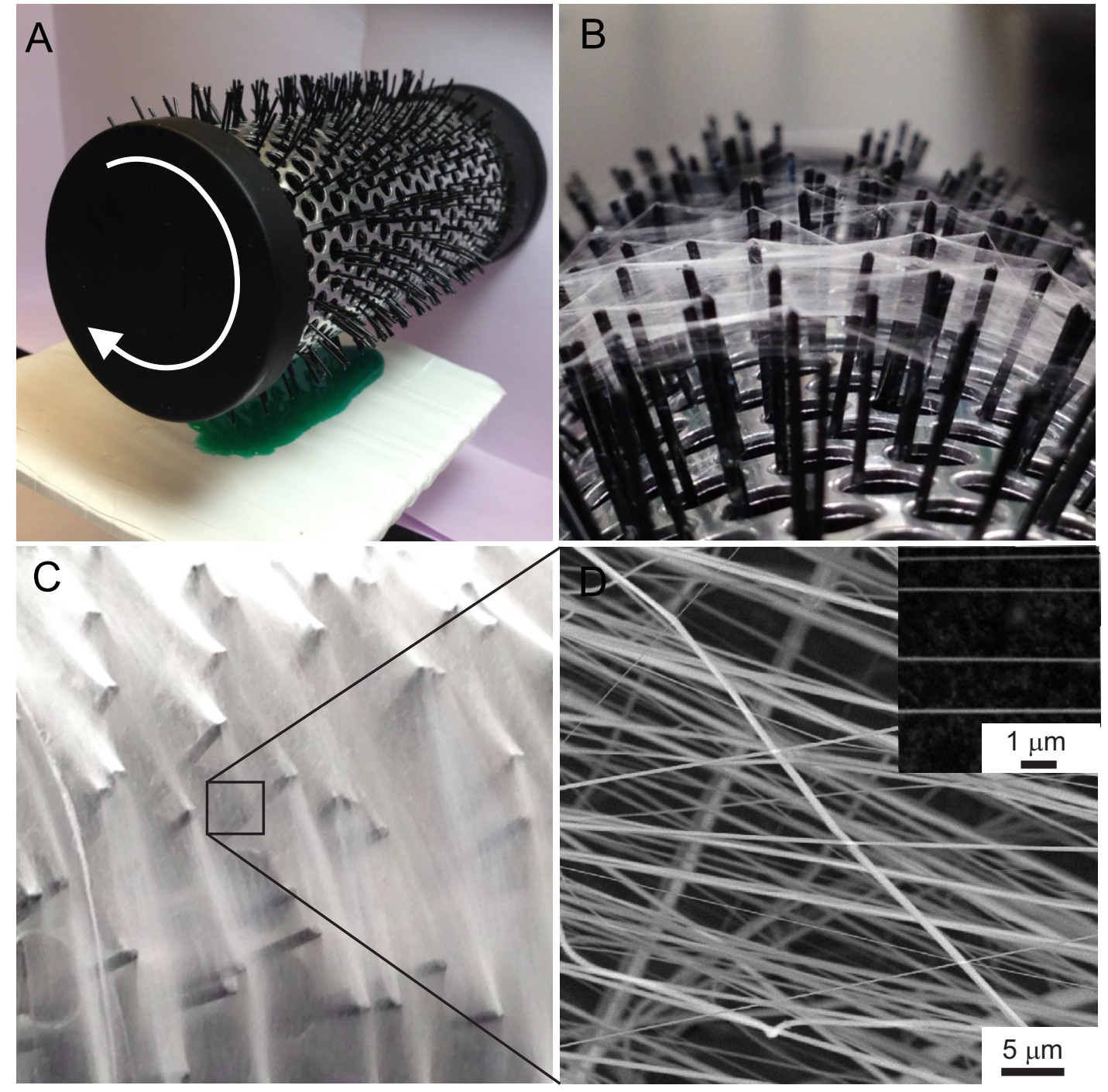

Fig. 3. Brush-spinning of nanofibers. (A) A round hairbrush is attached to a rotating motor and the PEO solution is poured onto a Teflon substrate. PEO solution is mixed with green dye for visualization purposes. (B) Nanofibers collected on the hairbrush rotated at 3000 RPM in 1 min and (C) in 5 min of spinning. (D) SEM image of brush-spun nanofibers.

Depending on the rotational mode of the spinning frame, the touch-spinning process enables both regular and random fiber meshes and continuous single free-standing nano and micro-fibers. For example, a rotating stage with a diameter of $5 \mathrm{~cm}$ was used to produce single free-standing $15 \mathrm{~cm}$ long polyacrylonitrile (PAN) micro and PCL nanofibers. Deviation of the fiber diameter along their length and between different batches is in the range 3-5\%. The Young's modulus of PCL fibers with diameters from 650 to $800 \mathrm{~nm}$ was measured to be $0.26 \pm 0.08 \mathrm{GPa}$, using a three-point bending test and an Atomic Force Microscope (AFM) (detailed procedure described in SM). Such results are comparable with the mechanical properties of PCL fibers produced by other common methods (25). 

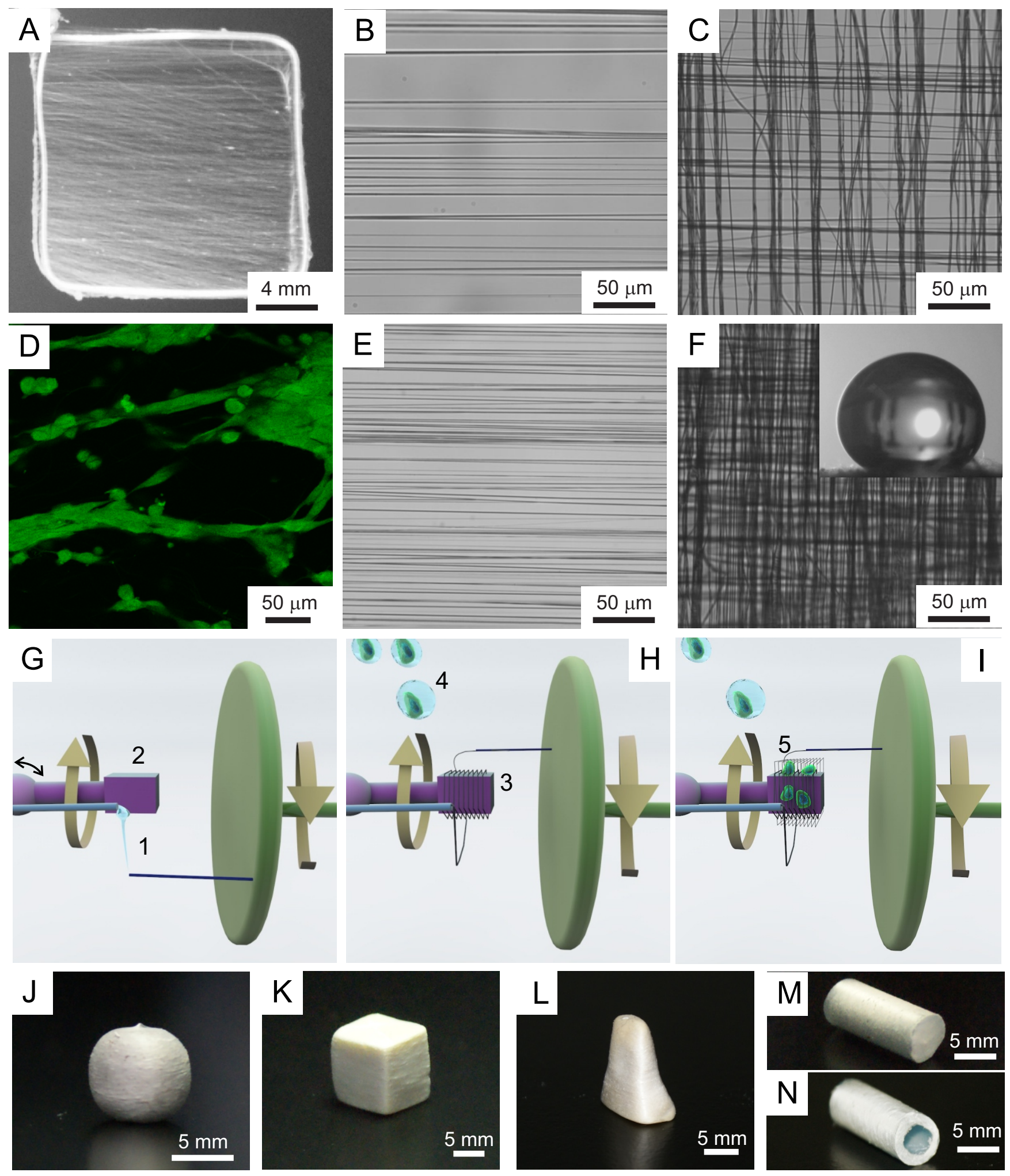

Fig. 4. Nanofiber meshes with controlled mesh size for tissue engineering and filtration applications. (A) A $15 \times 15 \mathrm{~mm}$ metallic frame size is placed behind the touch-spinning needle and fibers are collected on the frame. (B) Optical microscopy image of fibers on the frame produced after 2 minutes of touch-spinning. (C) After 2 minutes the frame was rotated by $90^{\circ}$ and touchspinning was continued for 2 further minutes resulting in a $28 \pm 7 \mu \mathrm{m}$ square mesh. (D) Confocal image of the mouse breast cancer cell grown on the mesh. (E) Optical microscopy image of fibers on the frame produced after 4 minutes of touch-spinning. (F) After 4 minutes the frame was rotated by 
$90^{\circ}$ and touch-spinning was continued for 2 further minutes resulting in a $5.8 \pm 1 \mu \mathrm{m}$ size mesh. Inset shows water droplet on the mesh of Teflon nanofibers (contact angle $\left.=154^{\circ}\right)$. $(\mathbf{G}-\mathbf{I})$ Preparation of 3D scaffolds by touch-spinning and simultaneous spraying of cells: (G) The fiber drawn by the rod (1) is wound onto the frame (2) of a desired shape (a cuboid for example) attached to the spool. The spool can be tilted at any angle to wind fibers onto frames with complicated geometries. $(\mathrm{H})$ Fibers are wound onto the frame with a controlled density (3) that can be regulated by the motion of the spool that shuttles back and forward. NIH-3T3 mouse fibroblast cells (4) are sprayed onto the frame simultaneously with winding of fibers. (I) 3D scaffold is made of the highly cellularized fiber meshes (5). (J-N) Different shapes and sizes of 3D-scaffolds obtained by winding nanofibers onto supporting frames mounted on a spool.

The essential difference of the touch-spinning method from other commonly used methods of nanofiber fabrication is in the mechanical control and manipulation of nanofibers that it offers. In contrast to all other methods, in touch-spinning single filament nanofibers are drawn by the mechanical force that determines not only fiber stretching but also guides fibers onto the spool providing better control over fiber alignment. For example, considering applications of nanofibers for tissue engineering scaffolds $(14,15)$, touch-spinning offers a very fast and practical method to produce scaffolds for cells with controlled mesh size in virtually any laboratory facility with no special requirement for equipment and training of personnel. For example, a supporting frame $15 \times 15 \mathrm{~mm}$ size was placed behind the touch-spinning needle and fibers were collected on the frame (Fig. 4A,B). After 2 minutes the frame was rotated by $90^{\circ}$ and touch-spinning was continued for 2 further minutes resulting in a $28 \pm 7 \mu \mathrm{m}$ square mesh (Fig. 4C). The mesh size can be controlled by adjusting the time of touch-spinning: 4 minutes of touch-spinning produced a mesh with size $5.8 \pm 1$ $\mu \mathrm{m}$ (Fig. 4E,F). The PCL meshes were successfully tested for scaffolding mouse breast cancer cells (Fig. 4D). Owing to its very simple set-up (Fig. 4G-I), the highly cellularized wet 3D-scaffolds (SM, Fig. S6) can be fabricated by combining fiber winding with simultaneous spraying of cells (26). The examples illustrated in Fig. 4J-N demonstrate the capability of the touch-spinning method for fabrication of biomimetic scaffolds on different scales from macroscopic shape and dimensions to microscopic fiber dimensions and alignment into various meshes that are relevant to mesh-like structures in human tissues.

Applications of the method developed are obviously not limited to tissue engineering scaffolds and extend to any other nanofiber application, for example filtration (27) when fibrous filters with a demanded mesh size can be prepared by simple winding of nanofibers as shown in Fig. 4A-C. Another obvious example is the fabrication of fibrous superhydrophobic coatings (28). Here, Teflon nanofiber meshes were tested for the fabrication of superhydrophobic surfaces (inset in Fig. 4F).

In this report, we describe a new method for drawing nanofibers. The method is based on a very simple and inexpensive set-up that does not require special training or skills. Using this method, nanofibers can be drawn in any non-specialized laboratory. It is possible to build a touch-spinning set-up by gluing a surface-modified glass rod to a rotating stage from which fibers can be spun from a free-liquid surface. A simple hairbrush can be used to scale up the fiber drawing to spin kilometers of nanofibers per minute. Owing to the set-up's simplicity and ability to manipulate nanofibers, 2D and 3D customized scaffolds of different dimensions, shapes, mesh sizes, fiber alignments and combinations with biological materials can be easily fabricated in minutes. 


\section{References}

1. J. R. Venugopal, S. Low, A. T. Choon, A. B. Kumar, S. Ramakrishna, Artificial Organs 32, 388-397 (2008).

2. S. Liao, B. Li, Z. Ma, H. Wei, C. Chan, S. Ramakrishna, Biomedical Materials 1, R45 (2006).

3. K. Ghanbari, S. Bathaie, M. Mousavi, Biosensors and Bioelectronics 23, 1825-1831 (2008).

4. T. Tamura, H. Kawakami, Nano Letters 10, 1324-1328 (2010).

5. S. Chen, H. Hou, F. Harnisch, S. A. Patil, A. A. Carmona-Martinez, S. Agarwal, Y. Zhang, S. Sinha-Ray, A. L. Yarin, A. Greiner, Energy \& Environmental Science 4, 1417-1421 (2011).

6. Z.-M. Huang, Y.-Z. Zhang, M. Kotaki, S. Ramakrishna, Composites Science and Technology 63, 2223-2253 (2003).

7. J. Huang, R. B. Kaner, Nature Materials 3, 783-786 (2004).

8. S. Ramakrishna, K. Fujihara, W.-E. Teo, T. Yong, Z. Ma, R. Ramaseshan, Materials Today 9, 40-50 (2006).

9. P. Gibson, H. Schreuder-Gibson, D. Rivin, Colloids and Surfaces A: Physicochemical and Engineering Aspects 187, 469-481 (2001).

10. K. Graham, M. Ouyang, T. Raether, T. Grafe, B. McDonald, P. Knauf, in Fifteenth Annual Technical Conference \& Expo of the American Filtration \& Separations Society, Galveston, Texas. (2002).

11. D. Li, J. Huang, R. B. Kaner, Accounts of Chemical Research 42, 135-145 (2008).

12. D. Li, Y. Xia, Advanced Materials 16, 1151-1170 (2004).

13. M. R. Williamson, R. Black, C. Kielty, Biomaterials 27, 3608-3616 (2006).

14. H. Yoshimoto, Y. Shin, H. Terai, J. Vacanti, Biomaterials 24, 2077-2082 (2003).

15. M. Shin, H. Yoshimoto, J. P. Vacanti, Tissue engineering 10, 33-41 (2004).

16. W. L. Grayson, P. H. G. Chao, D. Marolt, D. L. Kaplan, G. Vunjak-Novakovic, Trends Biotechnol. 26, 181-189 (2008).

17. V. L. Tsang, S. N. Bhatia, Adv. Drug Deliv. Rev. 56, 1635-1647 (2004).

18. T. Ondarcuhu, C. Joachim, Europhysics Letters 42, 215 (1998).

19. A. S. Nain, J. C. Wong, C. Amon, M. Sitti, Applied Physics Letters 89, 183105 (2006).

20. A. K. Kota, G. Kwon, A. Tuteja, NPG Asia Materials 6, e109 (2014).

21. P. Muthiah, S.-H. Hsu, W. Sigmund, Langmuir 26, 12483-12487 (2010).

22. R. Scheffler, N. S. Bell, W. Sigmund, Journal of Materials Research 25, 1595-1600 (2010).

23. X. Wang, T. Lin, Needleless electrospinning of nanofibers: Technology and applications. (CRC Press, 2013).

24. M. R. Badrossamay, H. A. McIlwee, J. A. Goss, K. K. Parker, Nano Letters 10, 2257-2261 (2010).

25. L. Sun, R. P. Han, J. Wang, C. Lim, Nanotechnology 19, 455706 (2008).

26. J. J. Stankus, J. J. Guan, K. Fujimoto, W. R. Wagner, Biomaterials 27, 735-744 (2006).

27. X. H. Qin, S. Y. Wang, Journal of Applied Polymer Science 102, 1285-1290 (2006).

28. A. Tuteja, W. Choi, M. Ma, J. M. Mabry, S. A. Mazzella, G. C. Rutledge, G. H. McKinley, R. E. Cohen, Science 318, 1618-1622 (2007). 